\title{
Unmanned Airship Design with Sliding Ballast: Modeling and Experimental Validation
}

\author{
Eric Lanteigne \\ Department of Mechanical Engineering \\ University of Ottawa \\ Ontario, Canada, K1N6N5 \\ Email: Eric.Lanteigne@uottawa.ca \\ Wail Gueaieb \\ School of Electrical Engineering and \\ Computer Science \\ University of Ottawa \\ Ontario, Canada, K1N6N5 \\ Email:wgueaieb@uottawa.ca
}

\author{
Dominic Robillard \\ and Steven Recoskie \\ Department of Mechanical Engineering \\ University of Ottawa \\ Ontario, Canada, K1N6N5
}

\begin{abstract}
Airships present many interesting opportunities for transport, surveillance and inspection but have seen little to no use in commercial or military unmanned applications. Generally underpowered, underactuated, and large in size, airships express difficulties in adverse atmospheric conditions or situations requiring rapid or precise maneuvers. In this paper, a miniature unmanned airship with a moving platform is presented to address the the limited altitude maneuverability of these vehicles. Simulated and experimental open-loop trajectories demonstrate that this architecture allows for large changes in vehicle pitch and, when combined with forward facing thrusters, rapid changes in altitude. Operational advantages such as increased hull rigidity and concentrated hardware inherent to the vehicle design are also discussed.
\end{abstract}

\section{INTRODUCTION}

Airships are well suited for use as reconnaissance vehicles because they can loiter, take off and land over short distances, and can sustain long endurance flights over long distances [1]. Conventional airships are driven by a set of actuators along the length of the hull with either elevators and rudders or thrusters for attitude and altitude control. Larger airships use air ballasts (also referred to as ballonets) for altitude and pitch control. For smaller vehicles, the typical actuator configuration is two thrusters oriented along the longitudinal axis for forward motion and a single thruster oriented along the vertical axis of the airship for altitude control [2]. Other configurations include thrusters in the longitudinal axis that can be rotated along the lateral axis [3]. With new light weight materials and manufacturing methods, many unconventional airship designs have been either proposed or tested. These vehicles have notable features that include the use of aerodynamic helium envelopes, multiple envelopes, segmented buoyancy cells, or utilize hybrid winged configurations to achieve lift [4-6]. A freight carrying vehicle based on the combination between quad-rotor and airship has been studied by Bestaoui to provide payload lift compensation [7]. A similar vehicle designed for transport is the discshaped airship with thrust vector control achieved with two engines on the lateral plane and one in front of the hull [8]. Theoretical designs have also been proposed such as an airship propelled by buoyancy forces alone [9]. It was shown that this type of vehicle could cruise long distances while consuming little energy by cycling the air between the bow and stern ballonets and exploiting the aerodynamics of the helium envelope to move forward.

Airships are typically underpowered and underactuated, and thus the controllability of the vehicle for autonomous flight in variable wind environments is critical for the safe and efficient operation [10]. More recently unmanned airships such as the fin-less airship Quanser MkII have used overactuation to address the controllability however the vehicle has been shown to be inherently unstable. As a consequence, the controller requires the use of optimization algorithms to determine the optimal combination of actuation inputs [11].

The autonomous airship architecture presented in this article provides an alternative to over-actuation and ballonets for the rapid altitude changes required when landing or avoiding obstacles. The proposed design is inspired by the concept of ballast control and center of gravity control (CG) in hydrodynamic or aerodynamic forced vehicles such as gliders [12], and is presented here to specifically examined the theoretical and experimental differences between altitude and pitch variations generated using aerodynamic control surfaces (elevator) versus configuration changes achieved by repositioning the gondola along the longitudinal axis of the helium envelope.

This remainder of this paper is organized as follows. In Section 2 we present the theoretical model of an airship with a moving platform using the method proposed by Gomes [13]. In Section 3 the design consideration of the proposed vehicle are outlined. In Section 4 we compare the simulated and experimental open-loop trajectories at various elevator and gondola positions, and in Section 5 final remarks are given.

\section{AIRSHIP MODELING}

In the following section, the dynamics of the airship are presented followed by the kinematic transformation between reference frames. 


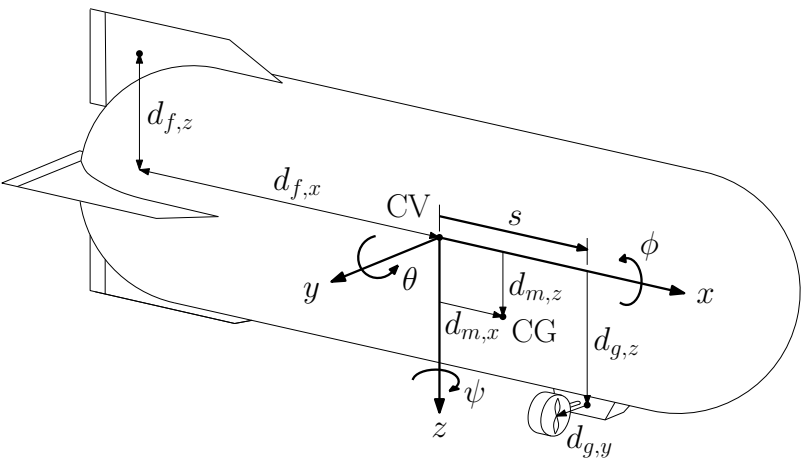

Fig. 1. Airship model

\section{A. Dynamics}

The dynamics of non-rigid airships are complex and nonlinear. In 2011, Li et al. [14] published a comprehensive review of airship dynamics spanning many decades. Since then, a significant amount of literature presents improvements to Newton-Euler equations $[15,16]$ or examines alternative approaches such as energy-based models [17, 18]. For simplicity, the non-linear dynamic model of the proposed airship was developed using Newton-Euler equations as derived by Gomes [13]. With reference to the coordinate system shown in Figure 1. the equation of motion is defined as,

$$
\mathbf{M} \dot{\mathbf{v}}=\mathbf{D}(\mathbf{v})+\mathbf{A}(\mathbf{v})+\mathbf{G}(\boldsymbol{\lambda})+\mathbf{U}
$$

where $\mathbf{M}$ is the mass matrix, $\mathbf{D}$ is the dynamics vector, A is the vector of aerodynamic components, $\mathbf{G}$ are the gravitational and buoyancy terms, $\mathbf{U}$ is the input force vector, $\boldsymbol{\lambda}$ is the directional cosine matrix, and $\mathbf{v}=\left[\mathbf{v}_{\mathbf{o}}{ }^{T} \boldsymbol{\omega}^{T}\right]^{T}$ is vector of linear velocities $\mathbf{v}_{\mathbf{o}}=\left[\begin{array}{lll}\dot{x} & \dot{y} & \dot{z}\end{array}\right]^{T}$ and angular

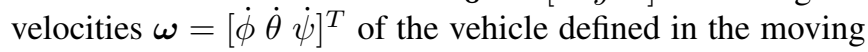
frame of reference located at the $\mathrm{CV}$ of the helium envelope.

After applying simplifications due to vehicle symmetry, the mass matrix is approximated by (2), where $\mathbf{d}_{\mathbf{C G}}$ is a skew symmetric matrix of the CG vector $\mathbf{r}_{\mathbf{C G}}=\left[\begin{array}{lll}d_{m, x} & 0 & d_{m, z}\end{array}\right]^{\prime}$ with respect to the CV. Since the position $s$ of the gondola is controllable, the components of $\mathbf{r}_{\mathbf{C G}}$ are given by $d_{m, x}=$ $s\left(m_{g} / m\right)$ and $d_{m, z}=\left(l_{R} m_{R}+l_{G} m_{G}\right) / m$. The added mass and inertia matrices $\mathbf{M}_{\mathbf{a}}$ and $\mathbf{J}_{\mathbf{a}}$ include the terms,

$$
\begin{gathered}
m_{x}=\left(1+k_{1}\right) m \\
m_{y}=m_{z}=\left(1+k_{2}\right) m \\
J_{x}=I_{f, x}+I_{g, x} \\
J_{y}=\left(1+k^{\prime}\right) I_{f, y}+I_{g, y} \\
J_{z}=\left(1+k^{\prime}\right) I_{f, z}+I_{g, z} \\
J_{x z}=I_{f, x z}+I_{g, x z}
\end{gathered}
$$

where the mass of the airship is equal to the sum of the mass of the gondola, the rail, and the envelope $m=m_{G}+m_{R}+$ $m_{E}$. The inertias of the fixed components as indicated by the subscript $f$ represent those of the envelope and rail and were determined from the computer-aided drafting (CAD) model of the airship. The gondola is approximated as a point mass its inertia is estimated using,

$$
\begin{gathered}
I_{g, x}=d_{g, z}^{2} \\
I_{g, y}=\sqrt{\left(s^{2}+d_{g, z}^{2}\right)} \\
I_{g, z}=s^{2} \\
I_{g, x z}=-s d_{g, z}
\end{gathered}
$$

The inertias of the gondola were not used in the computation of the virtual moment of inertia $J$ since the inertial contribution of the gondola increases from $5 \%$ to over $25 \%$ when the gondola position increases from $s=0 \mathrm{~m}$ to $s_{\max }=0.85 \mathrm{~m}$ (simulated maximum) and thus would overestimate the added mass effects of the displaced air.

Table I lists the geometric and physical properties of the simulated airship. The virtual mass terms $k_{1}, k_{2}$, and $k^{\prime}$ were approximated using the works of Lamb [19] and Munk [20].

TABLE I

SIMULATED AIRSHIP PHYSICAL PROPERTIES

\begin{tabular}{|c|l|c|}
\hline Symbol & Description & Value \\
\hline \hline$m_{E}$ & Envelope mass & $220 \mathrm{~g}$ \\
$m_{R}$ & Rail mass & $19 \mathrm{~g}$ \\
$m_{G}$ & Gondola mass & $121 \mathrm{~g}$ \\
$k_{1}$ & Lamb's inertia ratio about X & 0.1069 \\
$k_{2}$ & Lamb's inertia ratio about Y or Z & 0.8239 \\
$k^{\prime}$ & Lamb's inertia ratio about Y or Z & 0.5155 \\
$V$ & Airship volume & $0.311 \mathrm{~m}^{3}$ \\
$L$ & Airship length & $1.75 \mathrm{~m}$ \\
$D$ & Airship diameter & $0.5 \mathrm{~m}$ \\
$d_{g, z}$ & CV to gondola CG distance along z & $0.27 \mathrm{~m}$ \\
$d_{f, x}$ & CV to fin center distance along X & $0.8 \mathrm{~m}$ \\
$d_{f, z}$ & CV to fin center distance along $\mathrm{Z}$ & $0.27 \mathrm{~m}$ \\
\hline
\end{tabular}

The vehicle dynamics vector $\mathbf{D}(\mathbf{v})$ as described in [13] is given by,

$$
\mathbf{D}(\mathbf{v})=\left[\begin{array}{c}
\omega \times \mathbf{M}_{\mathbf{a}} \mathbf{v}_{\mathbf{o}}-m \omega \times\left(\omega \times \mathbf{r}_{\mathbf{C G}}\right) \\
\omega \times \mathbf{J}_{\mathbf{a}} \omega+m \mathbf{r}_{\mathbf{C G}} \times\left(\omega \times \mathbf{v}_{\mathbf{o}}\right)
\end{array}\right]
$$

The forces and moments in equation (13) are derived based on Newton's laws for rigid body motion about the CV in the body frame. These equations consist of both centrifugal $\left(\omega \times \mathbf{M}_{\mathbf{a}} \mathbf{v}_{\mathbf{o}}\right)$ and Coriolis $\left(\omega \times \mathbf{J}_{\mathbf{a}} \omega\right)$ components which are a function of the linear and angular velocities of the vehicle, and two additional terms resulting from the offset between the center of rotation and the center of mass.

The aerodynamics vector $\mathbf{A}$ acts to dampen linear and rotational rates of change in the vehicle. Many aerodynamic models in the literature are linear combinations of terms containing the angle of attack, the angle of sideslip, and the control surface deflections, all of which are multiplied by the square of the trimmed vehicle velocity. These models can be adequate for medium to high speed flight but have no effect at hover since aerodynamic dampening is reduced asymptotically with relative vehicle speed [21]. Gomes [13] and Ashraf et al. [16] present rotational pitch and yaw damping terms but neglect the envelope effects which are on the same order of magnitude as the fins [21]. 


$$
\mathbf{M}=\left[\begin{array}{cc}
\mathbf{M}_{\mathbf{a}} & m \mathbf{d}_{\mathbf{C G}}^{\mathbf{T}} \\
m \mathbf{d}_{\mathbf{C G}} & \mathbf{J}_{\mathbf{a}}
\end{array}\right]=\left[\begin{array}{cccccc}
m_{x} & 0 & 0 & 0 & m d_{m, z} & 0 \\
0 & m_{y} & 0 & -m d_{m, z} & 0 & m d_{m, x} \\
0 & 0 & m_{z} & 0 & -m d_{m, x} & 0 \\
0 & -m d_{m, z} & 0 & J_{x} & 0 & -J_{x z} \\
m d_{m, z} & 0 & -m d_{m, x} & 0 & J_{y} & 0 \\
0 & m d_{m, x} & 0 & J_{x z} & 0 & J_{z}
\end{array}\right]
$$

The components of the aerodynamic vector $\mathbf{A}(\mathbf{v})=$

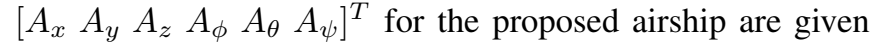
by (14) to (19), where the steady state dynamic pressure is defined as $q=1 / 2 \rho_{a} V^{2}$, the angles of attack and side slip relative to the air are defined as $\alpha=\arctan (\dot{z} / \dot{x})$ and $\beta=\arcsin (\dot{y} / V)$ with respect to the magnitude of the vehicle velocity $V=\sqrt{\dot{x}^{2}+\dot{y}^{2}+\dot{z}^{2}}$, and the elevator and rudder fin angles are defined as $\delta_{E}$ and $\delta_{R}$. These equations are based on the works of Mueller et al. [15] and Jones et al. [22] with additional rotational dampening terms (17) to (19), proportional to the square of the angular rates, included to account for the hull effects based on the work of Recoskie [21]. The magnitude of these terms is driven from the dynamic pressure which is negligible at trim flight speeds but becomes the primary aerodynamic dampening moments near hover.

The drag coefficients of the aerodynamic vector are given by (20) through (35) and the aerodynamic constants are described in Table II with references to where they can be determined for any airship using a combination of semiempirical models and geometric properties.

$$
\begin{gathered}
C_{X 1}=-\left[C_{D h o} S_{h}+C_{D f o} S_{f}+C_{D g o} S_{g}\right] \\
C_{X 2}=-C_{Y 1}=-C_{Z 1}=\left(k_{2}-k_{1}\right) \eta_{k} I_{1} S_{h} \\
C_{Y 2}=C_{Z 2}=-\frac{1}{2}\left(\frac{\delta C_{L}}{\delta \alpha}\right)_{f} S_{f} \eta_{f} \\
C_{Y 3}=-\left[C_{D c h} J_{1} S_{h}+C_{D c f} S_{f}+C_{D c g} S_{g}\right] \\
C_{Y 4}=C_{Z 4}=-\frac{1}{2}\left(\frac{\delta C_{L}}{\delta \alpha}\right)_{l} S_{f} \eta_{f} \\
C_{Z 3}=-\left[C_{D c h} J_{1} S_{h}+C_{D c f} S_{f}\right] \\
C_{\phi 1}=C_{D c g} S_{g} d_{g, z} \\
C_{\phi 2}=-2 C_{D c f} S_{f} d_{f, z}^{3} \\
C_{\phi 3}=-C_{D} c g * S_{g} d_{g, z} D^{2} \\
C_{\theta 1}=-C_{\psi 1}=\left(k_{2}-k_{1}\right) \eta_{k} I_{3} S_{h} L \\
C_{\theta 2}=-C_{\psi 2}=-\frac{1}{2}\left(\frac{\delta C_{L}}{\delta \alpha}\right)_{f} S_{f} \epsilon_{f} d_{f, x} \\
C_{\theta 3}=-\left[C_{D c h} J_{2} S_{h} L+C_{D c f} S_{f} d_{f, x}\right] \\
C_{\psi 3}=C_{D c h} J_{2} S_{h} L+C_{D c f} S_{f} d_{f, x}+C_{D c g} S_{g} s \\
C_{\theta 4}=-C_{\psi 4}=-\frac{1}{2}\left(\frac{\delta C_{L}}{\delta \delta_{E, R}}\right)_{l} S_{f} \epsilon_{f} d_{f, x} \\
C_{\theta 5}=-C_{D c f} S_{f} d_{f, x}^{3} \\
C_{\psi 5}=-\left[C_{D c f} S_{f} d_{f, x}^{3}-C_{D c g} S_{g} s^{3}\right]
\end{gathered}
$$

TABLE II

SIMULATED AIRSHIP AERODYNAMIC PROPERTIES

\begin{tabular}{|c|l|c|}
\hline Symbol & Description & Value \\
\hline \hline$C_{D h o}$ & Hull zero-incidence drag coefficient [23] & 0.024 \\
$C_{D f o}$ & Fin zero-incidence drag coefficient [23] & 0.003 \\
$C_{D g o}$ & Gondola zero-incidence drag coefficient & 0.01 \\
& [15] & \\
$C_{D c h}$ & Hull cross-flow drag coefficient [23] & 0.32 \\
$C_{D c f}$ & Fin cross-flow drag coefficient [24] & 2 \\
$C_{D c g}$ & Gondola cross-flow drag coefficient [15] & .25 \\
$\left(\frac{\delta C_{L}}{\delta \alpha}\right)_{f}$ & Derivative of fin lift coefficient with re- & 5.73 \\
$\left(\frac{\delta C_{L}}{\delta \delta_{E, R}}\right)_{f}$ & spect to angle of attack [24] & \\
$S_{h}$ & Derivative of fin lift coefficient with re- & 1.24 \\
$S_{f}$ & Hull reference area $V(2 / 3)$ & \\
$S_{g}$ & Fin reference area [5] & $0.46 \mathrm{~m}^{2}$ \\
$n_{f}$ & Gondola reference area [5] & $0.172 \mathrm{~m}^{2}$ \\
$n_{k}$ & Hull efficiency factor [22] & $0.0025 \mathrm{~m}^{2}$ \\
$I_{1}$ & & 0.4 \\
$I_{3}$ & Hull integrals [15] & 1.05 \\
$J_{1}$ & & 0 \\
$J_{2}$ & & -1.0839 \\
& & 1.7897 \\
\end{tabular}

The gravitational and buoyancy vector $\mathbf{G}$ is given by,

$$
\mathbf{G}(\boldsymbol{\lambda})=\left[\begin{array}{c}
m \mathbf{g} \\
m \mathbf{d}_{\mathbf{C G}} \mathbf{g}
\end{array}\right]-\left[\begin{array}{c}
\rho_{a} U \mathbf{g} \\
\rho_{a} U \mathbf{d}_{\mathbf{C G}} \mathbf{g}
\end{array}\right]
$$

where $U$ is the volume of the helium envelope. The term $\mathbf{g}=\boldsymbol{\lambda}^{T}\left[\begin{array}{ll}0 & 0\end{array}\right]^{T}$ is the gravitational vector expressed in the body frame using the directional cosine matrix $\lambda$, also known as the roll-pitch-yaw Euler sequence,

$$
\boldsymbol{\lambda}=\left[\begin{array}{ccc}
\mathrm{c} \theta \mathrm{c} \psi & \mathrm{s} \phi \mathrm{s} \theta \mathrm{c} \psi-\mathrm{c} \phi \mathrm{s} \psi & \mathrm{c} \phi \mathrm{s} \theta \mathrm{c} \psi+\mathrm{s} \phi \mathrm{s} \psi \\
\mathrm{c} \theta \mathrm{s} \psi & \mathrm{s} \phi \mathrm{s} \theta \mathrm{s} \psi+\mathrm{s} \phi \mathrm{s} \psi & \mathrm{c} \phi \mathrm{s} \theta \mathrm{s} \psi-\mathrm{c} \phi \mathrm{s} \psi \\
-\mathrm{s} \theta & \mathrm{s} \phi \mathrm{c} \theta & \mathrm{c} \phi \mathrm{c} \theta
\end{array}\right]
$$

Lastly, the input force vector $\mathbf{U}$ is given by,

$$
\mathbf{U}=\left[\begin{array}{c}
T_{R}+T_{L} \\
0 \\
0 \\
0 \\
\left(T_{R}+T_{L}\right) d_{g, z} \\
\left(T_{L}-T_{R}\right) d_{g, y}
\end{array}\right]
$$

where $T_{R}$ and $T_{L}$ are the right and left propeller thrust, and $d_{g, z}$ and $d_{g, y}$ are the normal distances from the right and left propeller centers to the $x-z$ plane as shown in Figure 1 .

\section{B. Kinematics}

The dynamic equation of motion (1) dictates how the vehicle accelerates based on the applied forces. Vehicle kinematics are applied in tandem to determine the vehicle's 


$$
\begin{gathered}
A_{x}=q\left[C_{X 1} \cos (\alpha)^{2} \cos (\beta)^{2}+C_{X 2}(\sin (2 \alpha) \sin (\alpha / 2)+\sin (2 \beta) \sin (\beta / 2))\right] \\
A_{y}=q\left(C_{Y 1} \cos (\beta / 2) \sin (2 \beta)+C_{Y 2} \sin (2 \beta)+C_{Y 3} \sin (\beta) \sin (|\beta|)+C_{Y 4}\left(2 \delta_{R}\right)\right) \\
A_{z}=q\left(C_{Z 1} \cos (\alpha / 2) \sin (2 \alpha)+C_{Z 2} \sin (2 \alpha)+C_{Z 3} \sin (\alpha) \sin (|\alpha|)+C_{Z 4}\left(2 \delta_{E}\right)\right) \\
A_{\phi}=q\left(C_{\phi 1} \sin (\beta) \sin (|\beta|)+\rho_{a} C_{\phi 3} \dot{\phi}|\dot{\phi}| / 2+\rho_{a} C_{\phi 3} \dot{\psi}|\dot{\psi}| / 2\right. \\
A_{\theta}=q\left(C_{M 1} \cos (\alpha / 2) \sin (2 \alpha)+C_{M 2} \sin (2 \alpha)+C_{M 3} \sin (\alpha) \sin (|\alpha|)+C_{M 4}\left(2 \delta_{E}\right)\right)+\rho_{a} C_{M 5} \dot{\theta}|\dot{\theta}| / 2 \\
A_{\psi}=q\left(C_{N 1} \cos (\beta / 2) \sin (2 \beta)+C_{N 2} \sin (2 \beta)+C_{N 3} \sin (\beta) \sin (|\beta|)+C_{N 4}\left(2 \delta_{R}\right)\right)+\rho_{a} C_{N 5} \dot{\psi}|\dot{\psi}| / 2
\end{gathered}
$$

velocity and position, and how they translate to the earth reference frame. The coordinates and trajectories in the earth reference frame can be determined from the equivalent states in the body reference frame premultipled by the matrix,

$$
\mathbf{v}_{g}=\left[\begin{array}{cc}
\lambda & 0_{3 \times 3} \\
0_{3 \times 3} & \mathbf{R}
\end{array}\right] \mathbf{v}+\mathbf{v}_{w}
$$

where $\mathbf{v}_{w}$ is the wind (steady + gusts) in a $6 \times 1$ vector and $\mathbf{R}$ is the rotation matrix described by:

$$
\mathbf{R}=\left[\begin{array}{ccc}
1 & \sin (\phi) \tan (\theta) & \cos (\phi) \tan (\theta) \\
0 & \cos (\phi) & -\sin (\phi) \\
0 & \sin (\phi) \sec (\theta) & \cos (\phi) \sec (\theta)
\end{array}\right]
$$

\section{Numerical simulations}

To illustrate the effects of modifying the gondola position, only the longitudinal motion of the airship is presented. The simulated trajectories were generated in an undisturbed environment with no wind, no temporal changes in pressure or temperature, and no other external disturbances. In addition, the thrusters were fixed to $T_{R}=T_{L}=T$ and the rudder angle was fixed to $\delta_{R U D}=0$ throughout the simulations. Only the air density as a function of the altitude was assumed to affect the buoyancy of the vehicle.

The open-loop dynamics were simulated to compare the flight characteristics of the airship when subjected to either full elevator input or gondola position changes with constant trust from both propellers from either a stationary initial position or with a constant initial horizontal velocity. For each simulation, the initial angular velocities were set to $\boldsymbol{\omega}=\mathbf{0}$ and the initial position of the CG was set to $\left[x_{g}, y_{g}, z_{g}\right]=[0,0,180] \mathrm{m}$. This corresponds to the neutrally buoyant altitude of the test area.

Two sets of trajectories were produced by simulating four different thruster levels. The simulated open-loop trajectories presented in Figure 2 show the first $4 \mathrm{~m}$ of horizontal motion assuming the airship has zero initial forward velocity $\mathbf{v}_{\mathbf{o}}=$ $\mathbf{0}$ at $t=0 \mathrm{~s}$. In this figure, the flight paths illustrated in black represent cases where the gondola position was set to $s=0.85 \mathrm{~m}$ with $\delta_{E}=0^{\circ}$, and the flight paths illustrated in gray represent cases where the gondola position was set to $s=0 \mathrm{~m}$ with $\delta_{E}=-20^{\circ}$.

The second set of trajectories, shown in Figure 3, illustrate the first $20 \mathrm{~m}$ of horizontal motion for the same airship configurations as above with the exception that the vehicle is traveling at an initial steady-state trim flight with $\mathbf{v}_{\mathbf{o}}=$

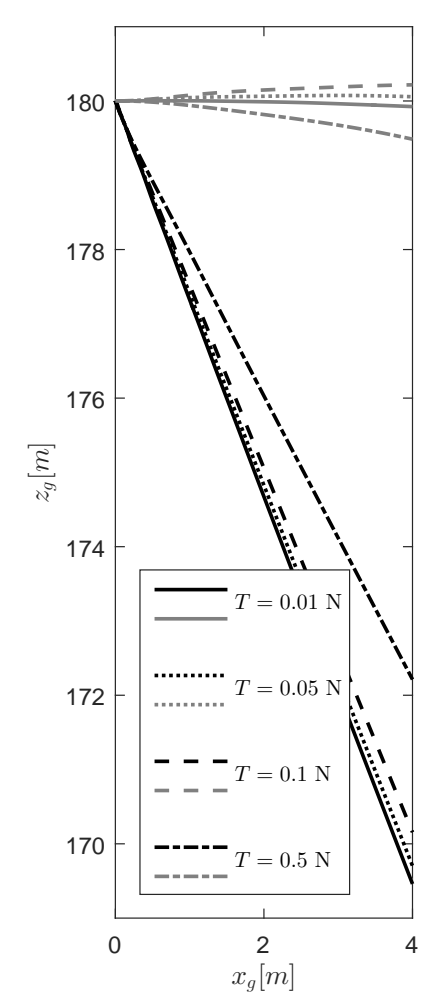

Fig. 2. First $4 \mathrm{~m}$ of horizontal displacement with zero initial velocity, where the trajectories in black represent the $s=0.85 \mathrm{~m}$ with $\delta_{E}=0^{\circ}$ configurations and the trajectories in gray represent the $s=0 \mathrm{~m}$ with $\delta_{E}=-20^{\circ}$ configurations.

$\left[\dot{x}_{s s}, 0,0\right]$ and $\theta=\dot{\theta}=0$ at $t=0 \mathrm{~s}$. For each case, the initial elevator angle was set to $\delta_{E}=0^{\circ}$ and the initial gondola position $s_{s s}$ was adjusted for level flight. As an example, in the case of $T=0.5 \mathrm{~N}$, the initial gondola position was $s_{s s}=0.227 \mathrm{~m}$. The simulated steady-state forward velocities and gondola positions for the four thruster levels are listed in Table III. The position of the gondola or the elevator is then gradually increased to $s=0.85 \mathrm{~m}$ or $\delta_{E}=-20^{\circ}$ over the first $3 \mathrm{~s}$ of the simulations. Similar to the first set of results, the flight paths illustrated in black represent cases where the gondola position was moved to $s=0.85 \mathrm{~m}$ at $t=3 \mathrm{~s}$, and the flight paths illustrated in gray represent cases where the elevator angle was moved to $\delta_{E}=-20^{\circ}$ at $t=3 \mathrm{~s}$. The trajectories in Figures 2 and 3 are presented with with equal axes to better illustrate the behavior of the vehicle. 


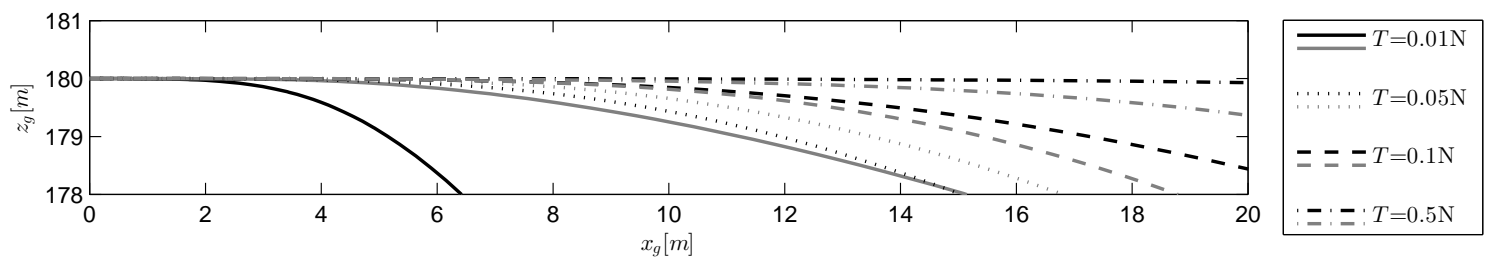

Fig. 3. First $20 \mathrm{~m}$ of horizontal displacement with a constant initial velocity, where the trajectories in black represent the $s=0.85 \mathrm{~m}$ with $\delta_{E}=0^{\circ}$ configurations and the trajectories in gray represent the $s=0 \mathrm{~m}$ with $\delta_{E}=-20^{\circ}$ configurations.

TABLE III

SIMULATED STEADY-STATE VELOCITIES AND GONDOLA POSITIONS FOR TRIM FLIGHT AT $t=0 \mathrm{~s}$

\begin{tabular}{|l||l|l|l|l|}
\hline$T[\mathrm{~N}]$ & 0.01 & 0.05 & 0.1 & 0.5 \\
\hline$\dot{x}_{s s}[\mathrm{~m} / \mathrm{s}]$ & 1.72 & 3.86 & 5.45 & 12.19 \\
$s_{s s}[\mathrm{~m}]$ & 0.005 & 0.023 & 0.045 & 0.227 \\
\hline
\end{tabular}

The simulated trajectories demonstrate that altitude changes can be initiated much more rapidly by changing the gondola position as opposed to the elevator at all thruster levels when starting from rest, and for all low thruster levels when traveling at a steady state initial velocity. The descent rate of the vehicle resulting from changes in the elevator angle only surpasses that resulting from changes in gondola position when the forward velocity of the vehicle is above $\dot{x}=3.75 \mathrm{~m} / \mathrm{s}$ as is the case in Figure 3 for trajectories generated with $T>0.05 \mathrm{~N}$. Moreover the descent trajectories at all truster levels are approximately linear when the gondola is at the foremost position when starting from a stationary position.

\section{EXPERIMENTAL AIRSHIP}

\section{A. Vehicle design}

A proof-of-concept airship was developed to validate the trajectories generated by the simulation and confirm the feasibility of the proposed model. The $3 \mathrm{D}$ printed gondola, shown in Figure 4, travels along a $4 \mathrm{~mm} \times 4 \mathrm{~mm} \times 1000 \mathrm{~mm}$ square carbon fiber keel embedded in a helium envelope. The envelope is fabricated from 2 Mil polyester polyurethane film with 90 Shore A hardness. Two forward facing thrusters provide a maximum of $T_{L}+T_{R}=0.15 \mathrm{~N}$ of thrust at full throttle and $0.05 \mathrm{~N}$ at half throttle. A gear motor is used for positioning the gondola along the length of the rod. The gondola is approximately $0.05 \mathrm{~m}$ long and can travel to the foremost position of $s=0.5 \mathrm{~m}$ at the bow of the airship and the rearmost position of $s=-0.45 \mathrm{~m}$ at the stern. Secondary ballast weights were added to balance the fin weight and ensure that $\theta=0^{\circ}$ when $s=0 \mathrm{~m}$ while stationary. The masses of the individual components of the vehicle are listed in Table IV.

The vehicle was equipped with a Nanowii flight controller running the Multiwii open-source firmware with a 3-axis gyroscopic sensor and a 3-axis accelerometer. State variables and commands are sent from a MatLab GUI to the controller

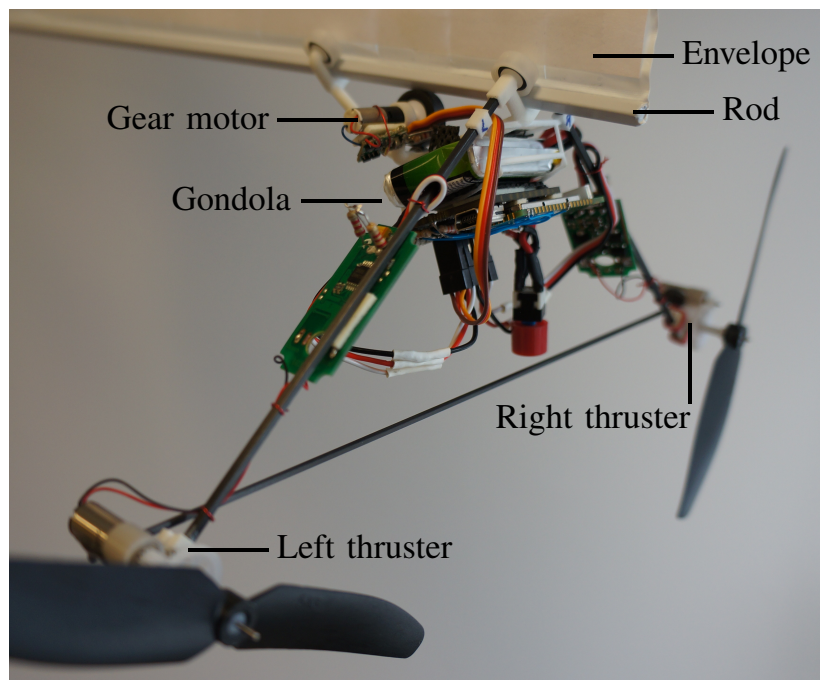

Fig. 4. Gondola architecture.

TABLE IV

EXPERIMENTAL AIRSHIP PHYSICAL PROPERTIES

\begin{tabular}{|c|l|c|}
\hline Symbol & Description & Value \\
\hline \hline$m_{E}+m_{R}$ & Envelope and rail mass & $230 \mathrm{~g}$ \\
$m_{G}$ & Gondola mass & $60 \mathrm{~g}$ \\
$m_{F}$ & Fin mass & $24 \mathrm{~g}$ \\
$m_{B}$ & Ballast mass & $52-105 \mathrm{~g}$ \\
$V$ & Airship volume & $0.39 \mathrm{~m}^{3}$ \\
$L$ & Airship length & $1.83 \mathrm{~m}$ \\
$D$ & Airship maximum diameter & $0.6 \mathrm{~m}$ \\
\hline
\end{tabular}

through a wireless serial interface using a long-range Bluetooth module.

\section{B. Open-loop trajectories}

The experimental open-loop trajectories were all generated with the vehicle in the stationary position $\mathbf{v}=\mathbf{0}$ at $t=0 \mathrm{~s}$. The altitude was normalize to $z_{g}=0 \mathrm{~m}$ for all measurements, and small fixed-ballast adjustments were made to account for changes in atmospheric pressure between tests.

The results shown in Figures 5 illustrate the mean trajectories for the first $3.5 \mathrm{~m}$ of flight with the gondola at the foremost position of $s=0.5 \mathrm{~m}$ with full and half throttle. The second set of results, shown in Figure 6, illustrate the mean trajectories at full throttle for five different gondola positions. At the foremost and rearmost gondola positions 


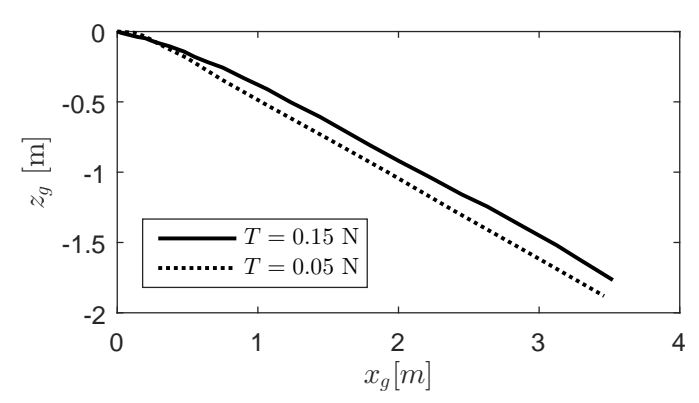

Fig. 5. Average experimental trajectories with $s=0.5 \mathrm{~m}$.

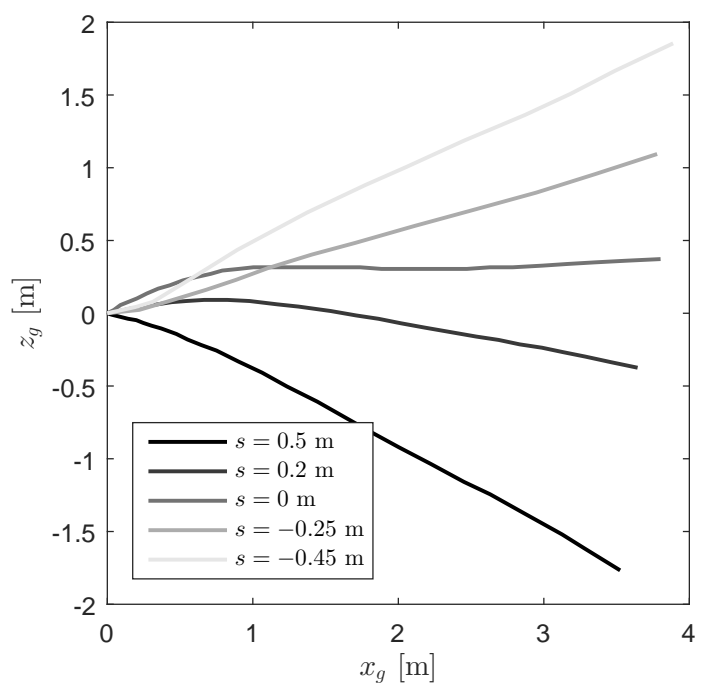

Fig. 6. Average experimental trajectories at full throttle, $\delta_{E}=0^{\circ}$, and various gondola positions.

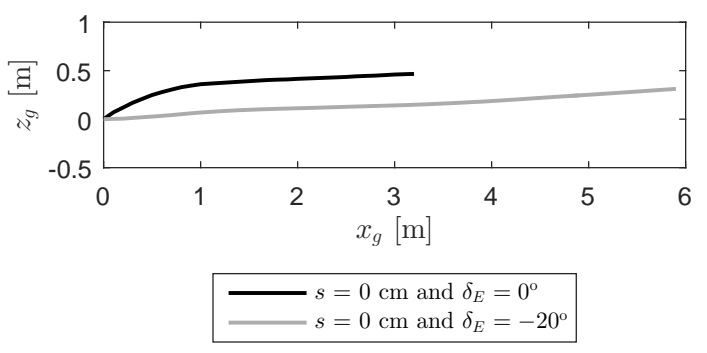

Fig. 7. Experimental trajectory at full throttle with elevator changes.

of $s=0.5 \mathrm{~m}$ and $s=-0.45 \mathrm{~m}$, the mean steady-state pitches are $-32^{\circ}$ and $27^{\circ}$ respectively. The results show that trajectories generated with at these positions demonstrated approximately linear flight paths after the first meter of horizontal motion.

Lastly, the mean experimental trajectory generated at full throttle with $\delta_{E}=-20^{\circ}$ and $s=0 \mathrm{~m}$ is shown along side the mean trajectory with $\delta_{E}=0^{\circ}$ and $s=0 \mathrm{~m}$ in Figure 7. Both the mean trajectory and all individual trials with $\delta_{E}=-20^{\circ}$ and $s=0 \mathrm{~m}$ exhibited upward motion in the first $6.5 \mathrm{~m}$ of flight when starting from a stationary position, and reached
TABLE V

STANDARD DEVIATION OF THE VERTICAL DISPLACEMENT FOR THE FIRST 3.5 M OF FLIGHT

\begin{tabular}{|c|c|c|c|}
\hline$s[\mathrm{~m}]$ & $\delta_{E}[\mathrm{deg}]$ & $\sigma[\mathrm{m}]$ & $\sigma_{p}[\mathrm{~m}]$ \\
\hline \hline 0.5 & 0 & 0.043 & 0.034 \\
0.2 & 0 & 0.049 & 0.051 \\
0 & 0 & 0178 & 0.181 \\
0 & 20 & 0.111 & 0.071 \\
\hline
\end{tabular}

a maximum average velocity of $1.5 \mathrm{~m} / \mathrm{s}$. This behavior is similar to the simulation results shown in Figure 2, where the model only begins to descend once a horizontal velocity of $2.3 \mathrm{~m} / \mathrm{s}$ is reached. A longer test area would have been required to reach the steady-state velocity of the vehicle and evaluate the effectiveness of the elevators.

\section{DISCUSSION}

The moment and product of inertia estimates are known to have considerable error and may have contributed to the discrepancy between the simulated trajectories and the actual paths [25]. Nonetheless, the model predicts that the principal moment of inertia about the $\theta$ axis increase by $2 \%$ at $s=0.2 \mathrm{~m}$ to $11 \%$ at $s=0.45 \mathrm{~m}$. Increasing the moment of inertia effectively attenuates the effects of external disturbances on the pitch of the vehicle and, when combined with forward motion, on the vertical displacement. The effect was observed in the experimental open-loop trajectories as demonstrated by the aggregate results in Table V. The standard deviation $\sigma$ and the pooled standard deviation $\sigma_{p}$ for the first $3.5 \mathrm{~m}$ of flight of four combinations of $s$ and $\delta_{E}$ illustrate that trajectories generated with $s=0 \mathrm{~m}$ exhibited the greatest variability in vertical displacement whereas both simulated and experimental trajectories with large values of $s$ demonstrated approximately linear flight paths when starting from rest. The composite image in Figure 8 further illustrates the linearity of the motion. The individual images in Figure 8 were taken at $1 \mathrm{~s}$ intervals and represent one of the sample trajectories used to generate the average trajectory with $s=0.5 \mathrm{~m}$ shown in Figures 5 .

The linear behavior is only valid near the neutrally buoyant reference altitude after which the difference between the gravity and buoyancy vectors dominates the dynamics of the vehicle and the motion become oscillatory as illustrated by the simulated trajectory in Figure 9. The simulated trajectory with $T=0.1 \mathrm{~N}$ was approximately linear for the first 100 $\mathrm{m}$ of horizontal motion then oscillates until a steady state altitude of approximately $150 \mathrm{~m}$ below sea level is reached. The large drop in altitude exhibited by the model is due to the assumption of constant trust. In reality, the propeller thrust is proportional to the relative vehicle speed and dependent on the battery capacity. A thruster model should be implemented to better correlate the simulated and experimental velocities.

The mass and moments of inertia of the fins were neglected in the simulated airship but were found to significantly alter the CG of the airship and reduce the pitch angle at $s_{\max }$. In addition, the simulated vehicle shape was also simplified and did not reflect the progressive reduction in the cross section 


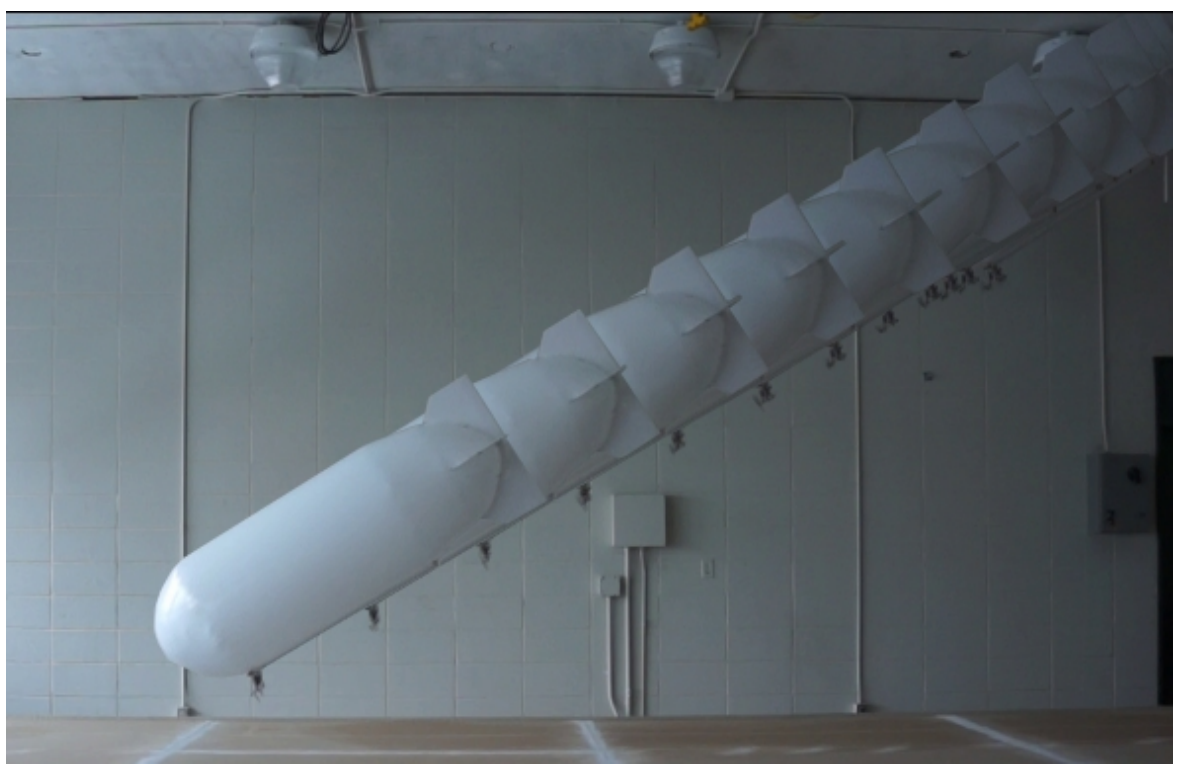

Fig. 8. Composite image of the airship motion with $s=0.5 \mathrm{~m}$.

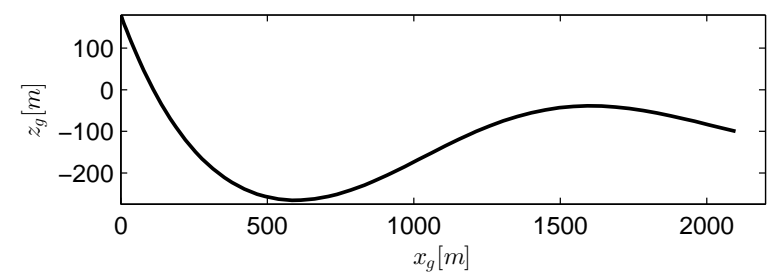

Fig. 9. Simulated trajectory with $T=0.1 \mathrm{~N}$ and zero initial velocity.

of the helium envelope at the bow of the vehicle. Future iterations of the design and dynamic model will include these feature to better predict the motion of the vehicle. Wind tunnel testing should also be performed to accurately model the drag of the vehicle.

Other considerations not evident from the results is the rigidity imparted by the keel-envelope combination. Maintaining the envelope shape is an important design consideration for non-rigid and semi-rigid airships since the envelop is in opposition to bending and shear forces resulting from the pressure of the lifting gas and all dynamic and aerodynamic loads under all operating conditions [5]. Bennaceur et al. presented a Euler-Lagrange airship model that allows for aeroelastic deformations of the hull and concluded that the impact of flexibility in the dynamics should not be neglected [26]. The high rigidity of the carbon fiber keel decreased the overall flexibility of the vehicle and, if the envelope was partially deflated, maintained the cylindrical form of the envelope and the gondola orientation with respect to the vehicle.

Lastly, when the gondola was in the foremost position, the vehicle was more easily manipulated. This configuration could potentially facilitate autonomous landings or payload deliveries in larger vehicles by ensuring that the gondola reaches the ground before the envelope.

\section{CONClusion AND Future Work}

This paper has demonstrated that repositioning the gondola and primary ballast of unmanned airships along the longitudinal axis significantly alters the pitch of the vehicle thereby minimizing the aerodynamic drag in a wide range of inclinations and allowing for rapid changes in altitude.

Both simulation and experimental open-loop flight trajectories have shown near linear motions at large pitch angles around the neutrally buoyant reference altitude. The ability to rapidly change altitude addresses one of the major limitations of unmanned airships: accurate and rapid landing. These results are significant since also they demonstrate that the velocity and rate of descent are, to a certain extent, independent. This offers greater flexibility in the face of adverse conditions or precise landing requirements.

The proposed architecture also features numerous operational advantages such as increased hull rigidity in the face of envelop decompression and concentrated hardware. These considerations have significant implications in design of commercially viable unmanned airships. Future design iterations will include a more robust method for determining the gondola position and including thrust vectoring in the lateral axis of the actuator. These developments would also require a more robust geometric model and mass estimation, as well as a propeller model for accurately estimating the thrust.

\section{ACKNOWLEDGMENT}

This work was supported by NSERC Discovery grant RGPIN-2014-04501

\section{REFERENCES}

[1] J. Rao, Z. Gong, J. Luo, and S. Xie, "Unmanned airships for emergency management," in IEEE International Workshop on Safety, Security and Rescue Robotics, June 2005, pp. 125-130. 
[2] M. Frye, S. Gammon, and C. Qian, "The 6-DOF dynamic model and simulation of the tri-turbofan remotecontrolled airship," in American Control Conference, July 2007, pp. 816-821.

[3] J. Ko, D. J. Klein, D. Fox, and D. Haehnel, "Gaussian processes and reinforcement learning for identification and control of an autonomous blimp," in IEEE International Conference on Robotics and Automation, April 2007, pp. 742-747.

[4] B. H. Kroeplin, "Segmented aircraft comprising an energy medium," 12 2011, US Patent App. 13/003,638.

[5] G. A. Khoury, Airship technology. Cambridge University Press, 2012.

[6] M. Y. Guanxiong Li, Dongli Ma, "Research of near space hybrid power airship with a novel method of energy storage," International Journal of Hydrogen Energy, vol. 40, pp. 9555-9562, August 2015.

[7] Y. Bestaoui, "Modeling of a quad-rotor airship with wind and varying freight mass effects," International Review of Aerospace Engineering, vol. 2, no. 2, pp. 9197, 2009.

[8] M. Madonia, A. Di Furia, S. Bonasia, and D. Vucinic, "Structural analysis of an engine chassis for a discshaped airship with thrust vector control," SAE International Journal of Materials and Manufacturing, vol. 8, no. 1, pp. 128-138, 2015.

[9] C. Wu, C. H. Moog, and Y. Hu, "Modelling and linear control of a buoyancy-driven airship," in Asian Control Conference, August 2009, pp. 75-80.

[10] S. Saripalli, J. Montgomery, and G. Sukhatme, "Visually guided landing of an unmanned aerial vehicle," IEEE Transactions on Robotics and Automation, vol. 19, no. 3, pp. 371-380, 2003.

[11] T. Liesk, M. Nahon, and B. Boulet, "Design and experimental validation of a nonlinear low-level controller for an unmanned fin-less airship," IEEE Transactions on Control Systems Technology, vol. 21, no. 1, pp. 149161, Jan 2013.

[12] P. Bhatta and N. L. Leonard, "Nonlinear gliding stability and control for vehicles with hydrodynamic forcing," Automatica, vol. 44, no. 5, pp. 1240- 1250, 2008.

[13] S. B. V. Gomes, "An investigation into the flight dynamics of airships with application to the yez-2a," Ph.D. dissertation, Cranfield University, 1990.

[14] Y. Li, M. Nahon, and I. Sharf, "Airship dynamics modeling: A literature review," Progress in Aerospace Sciences, vol. 47, no. 3, pp. 217-239, 2011.

[15] J. B. Mueller, M. A. Paluszek, and Y. Zhao, "Development of an aerodynamic model and control law design for a high altitude airship," in AIAA 3rd Unmanned Unlimited Technical Conference, 2004, pp. 1-17.

[16] M. Ashraf and M. Choudhry, "Dynamic modeling of the airship with matlab using geometrical aerodynamic parameters," Aerospace Science and Technology, vol. 25 , no. 1, pp. 56-64, 2013.

[17] P. G. Thomasson, "Equations of motion of a vehicle in a moving fluid," Journal of Aircraft, vol. 37, no. 4, pp. 630-639, 2000.

[18] Y. B. Sebbane, Lighter than Air Robots. Springer Netherlands, 2012, vol. 58.

[19] H. Lamb, "The inertia coefficients of an ellipsoid moving in fluid," Aeronautical Research Committee, Tech. Rep., 1918.

[20] M. M. Munk, “Aerodynamics of airships," Aerodynamic Theory, vol. 6, pp. 32-48, 1936.

[21] S. Recoskie, "Autonomous hybrid powered long ranged airship for surveillance and guidance," Ph.D. dissertation, University of Ottawa, 2014.

[22] S. P. Jones and J. D. DeLaurier, "Aerodynamic estimation techniques for aerostats and airships," Journal of Aircraft, vol. 20, no. 2, pp. 120-126, 1983.

[23] S. F. Hoerner, Fluid dynamic Drag. Hoerner Fluid Dynamics, 1993.

[24] D. P. Raymer, Aircraft Design: A Conceptual Approach (Aiaa Education Series). Amer Institute of Aeronautics, 2012.

[25] W. Yongmei, Z. Ming, Z. Zongyu, and Z. Zewei, "Trajectory tracking of a high altitude unmanned airship based on adaptive feedback linearization," in International Conference on Mechatronic Science, Electric Engineering and Computer (MEC), August 2011, pp. 2257-2261.

[26] S. Bennaceur and N. Azouz, "Contribution of the added masses in the dynamic modelling of flexible airships," Nonlinear Dynamics, vol. 67, no. 1, pp. 215-226, 2012. 